\title{
What do we need to transfer pharmacogenetics findings into the clinic?
}

\author{
Elise MA Slob1, Susanne JH Vijverberg ${ }^{1}$, Mariëlle W Pijnenburg ${ }^{2}$, Gerard H Koppelman ${ }^{3,4}$ \& \\ Anke-Hilse Maitland-van der Zee*,1 \\ ${ }^{1}$ Department of Respiratory Medicine, Academic Medical Center, University of Amsterdam, P.O. Box 22700, NL-1100 DE \\ Amsterdam, The Netherlands \\ ${ }^{2}$ Department of Paediatrics, Paediatric Pulmonology \& Allergology, Erasmus Medical Center-Sophia Children's Hospital, Rotterdam, \\ The Netherlands \\ ${ }^{3}$ Department of Paediatric Pulmonology \& Paediatric Allergology, University of Groningen, University Medical Center Groningen, \\ Beatrix Children's Hospital, Groningen, The Netherlands \\ ${ }^{4}$ Groningen Research Institute for Asthma \& COPD (GRIAC), University of Groningen, University Medical Center Groningen, \\ Groningen, The Netherlands \\ *Author for correspondence: Tel.: +31 20566 4356; Fax: +31 20566 9001; a.h.maitland@amc.nl
}

\section{"With new rapid point-of-care tests coming to the clinics, the way is paved to further implement pharmacogenetics into usual care."}

First draft submitted: 26 February 2018; Accepted for publication: 2 March 2018; Published online: 27 April 2018

Keywords: infrastructure $\bullet$ implementation $\bullet$ pharmacogenetics $\bullet$ precision medicine

Recently, a portable USB compatible handheld DNA sequencer became commercially available to genotype individuals without need of a laboratory [1]. However, does the rise of point-of-care testing imply that we are ready to implement pharmacogenetics into daily practice? Pharmacogenetics can help in defining optimal drug dosage, identifying patients at risk of drug-induced toxicity or adverse drug effects and predicting whether a prescribed drug will be effective [2]. In an ideal world, individual genetic information should be integrated in computer provider order entry (CPOE) systems and/or clinical decision support tools to calculate precise dosing regimens or to establish effectivity or risks of treatment before physicians generate electronic prescriptions. But what do we need to reach this stage?

First, evidence is key in implementation of pharmacogenetics testing in clinical practice, yet one can argue how much evidence is 'enough' to convince stakeholders. According to the clinical evidence pyramid, most weight is given to randomized controlled trials (RCTs) that compare pharmacogenetic-guided treatment strategy to usual care. However, these trials are often expensive and not always feasible to perform due to small patient populations in certain diseases. Furthermore, an RCT can even be considered unethical. For example, in case of strong observational evidence of genetic variants associated with life-threatening drug-induced toxicities [3], such as CYP2D6 genotype of breastfeeding mothers and codeine-induced neonate mortality [4]. Thus, it is important to reach consensus what level of evidence is needed to change clinical practice when precision medicine RCTs are not feasible or ethical. In our opinion strong observational evidence should be sufficient in some cases, when further randomized studies are deemed not feasible and/or ethical.

The request for precision medicine trials to gather clinical evidence is nicely illustrated by long-acting $\beta$-agonists (LABA) pharmacogenomics. These drugs are used in asthma treatment to relieve bronchoconstriction. An SNP in the $A D R B 2$ gene - encoding the beta2-adrenergic receptor - has been associated with poor response to LABA in children. The variant (rs1042713) leads to a change in amino acids of the receptor at position 16 (glycine into arginine), and is also known as Arg16. A large meta-analysis of observational studies [5] as well as a genotypestratified trial [6] showed that children carrying the variant have poor response to LABA and would benefit from alternative treatment. Despite this evidence, there is no movement toward genotyping before treatment with LABA yet. A precision medicine RCT is currently ongoing to compare $A D R B 2$-guided treatment to usual care [7] in order to change clinical practice in case of positive results. 
In addition to gathering 'enough' evidence and reporting effect measures, it is important that measures of clinical validity are reported in pharmacogenetic studies. Clinical validity and utility outcome measures as population impact measures (number needed to treat, number needed to genotype) and predictive values of utility (positive predictive value, negative predictive value) need to be evaluated [8]. This is important to interpret pharmacogenetics results for use in clinical practice. However, often pharmacogenetic studies only report measures of association.

Another important outcome measure is cost-effectiveness. Before society is willing to pay for pharmacogenetic tests, it should be assessed whether implementation of such tests are worth the money. Do they lead to an important difference in the patient's health, quality of life and healthcare resource utilization? What is the price of the test, and what are the costs per quality adjusted life year? Only if these costs are under a certain threshold, genetic testing will be implemented in standard clinical care [9].

Furthermore, a proper infrastructure is one of the other key conditions that is required to implement pharmacogenetics into clinical practice. A good infrastructure relies on sufficient facilities for genotyping, involvement of all stakeholders and education for healthcare providers. One of the current ongoing studies to optimize infrastructure is the PREPARE trial [10]. This is a prospective, European RCT across multiple genes, multiple drugs, multiple ethnicities and multiple healthcare systems conducted by the Ubiquitous Pharmacogenomics (U-PGx) consortium. In the PREPARE trial, a panel of 50 variants will be tested covering 13 'pharmacogenes'. These variants have clinical consequence regarding drug and dose selection of the 41 drugs for which genotyping guidelines already exist [11]. These drugs will be started in patients randomized to a pharmacogenetics and a usual care arm. Adverse events will be documented as a primary end point for at least 12 weeks up to a maximum of 18 months. A decrease of the adverse events and increase in effectiveness is expected in the pharmacogenetics arm compared with the usual care arm [10]. It is expected that this project will facilitate pharmacogenomics testing in clinical practice in Europe.

To transfer pharmacogenetic findings to the clinic, it is important that the healthcare framework contains facilities to have the patient's genotype information available in electronic healthcare records at the time of prescribing or dispensing a drug [12]. Currently, this is often missing. Pharmacogenetic testing is still reactive (testing specific pharmacogenetic variants to explain adverse effects or poor response) instead of preemptive (screening panel of pharmacogenetic variants in an early stage) [13]. This preemptive approach is also used in the PREPARE trial. But at which moment in time should such a screening be offered and be cost effective: in elderly, polypharmacy patients with high risk of using drugs of interest? Or should it be offered in early adulthood before prescription of any drugs of interest? We can save pharmacogenetic information for the rest of a patients' life, but we need to take care of protection of these genetic data to prevent it from unethical or commercial usage and also consider storage requirements [2].

A remaining question is: where in the healthcare system should genotyping be placed? This depends on the infrastructure and the velocity that is required to obtain a genotyping result. We should consider using rapid point-of-care tests for genotyping variants that are going to be commonly requested and where rapid outcomes are required. For example, coumarin genotyping outcomes should ideally be available within 1 day. In the EU-PACT trial, rapid point-of-care coumarin testing was used successfully [14]. The earlier mentioned DNA sequencer might fulfill this demand [1].

To make implementation of pharmacogenetics into the clinic a success, many stakeholders need to be involved: patients and their families, healthcare professionals, authorities, academic institutions, disease-centric organizations, healthcare insurance companies, developers of genome analyzers and scientists. All parties should be accurately informed about possibilities and limitations of genotyping for drug response [15,16]. As shared decision making is considered to become the standard, we must provide the patient insight in their treatment plan. The benefit-harm charts, for example, show different combinations of outcome risks in low-dose aspirin treatment for primary prevention of cardiovascular disease to the patients. They can help to make individual treatment decisions [17]. These charts are a good example of involving the patient and should inspire future precision medicine guideline developers.

If we want to actively involve healthcare professionals and patients in pharmacogenetics, we should invest in education on genotype-drug relations and the interpretation of genotyping outcomes. Studies show that the majority of physicians and other healthcare providers currently do not feel confident using pharmacogenetic knowledge in daily practice [12]. Pharmacogenetic courses are increasingly included in curricula of medical and pharmaceutical education programs at universities. However, understandable information for practicing health care providers and patients on how to interpret pharmacogenetic test results is important. In order to stimulate education, the U-PGx 
consortium provides e-learning modules on their website and education days to increase knowledge of healthcare professionals and patients [10].

Furthermore, to facilitate healthcare providers to use pharmacogenetics in daily practice, we need to integrate pharmacogenetic-prescribing tools in the prescribing system and pharmacogenetic guidelines need to be widely available [16]. In The Netherlands, The Royal Dutch Society for Pharmacy (KNMP) defined in guidelines how to bring pharmacogenetics results into dosing advices and updates these advices at least every 4 years. Computer provider order entry systems and clinical decision support tools in Dutch hospitals and pharmacies are able to integrate these advices to help pharmacists and doctors in precision dosing [18]. This should be further developed for worldwide use. The U-PGx consortium is taking another step forward by translating these advices into Greek, Slovenian, English, German, Spanish and Italian [10]. This hopefully leads to rapid implementation in these countries.

To conclude, we are moving onward towards implementation of pharmacogenetics into clinical practice. Corner stones in future steps should be evidence and infrastructure. New evidence must include sufficient outcome measures focusing on clinical validity, utility and cost-effectiveness. The infrastructure should contain sufficient facilities for genotyping, involvement of all stakeholders and education for healthcare providers. With new rapid point-of-care tests coming to the clinics, the way is paved to further implement pharmacogenetics into usual care.

Financial \& competing interests disclosure

The authors have no relevant affiliations or financial involvement with any organization or entity with a financial interest in or financial conflict with the subject matter or materials discussed in the manuscript. This includes employment, consultancies, honoraria, stock ownership or options, expert testimony, grants or patents received or pending, or royalties.

No writing assistance was utilized in the production of this manuscript.

\section{References}

1 Jain M, Koren S, Miga H et al. Nanopore sequencing and assembly of a human genome with ultra-long reads. Nat. Biotechnol. 36(4), 338-345 (2018).

2 Kapoor R, Tan-Koi WC, Teo YY. Role of pharmacogenetics in public health and clinical health care: a SWOT analysis. Eur. J. Hum. Genet. 24(12), 1651-1657 (2016).

3 Maagdenberg H, Vijverberg SJ, Bierings MB et al. Pharmacogenomics in pediatric patients: towards personalized medicine. Paediatr. Drugs 18(4), 251-260 (2016).

4 Koren G, Cairns J, Chitayat D, Gaedigk A, Leeder SJ. Pharmacogenetics of morphine poisoning in a breastfed neonate of a codeine-prescribed mother. Lancet 368(9536), 704 (2006).

5 Turner S, Francis B, Vijverberg S et al. Childhood asthma exacerbations and the Arg16 beta2-receptor polymorphism: a meta-analysis stratified by treatment. J. Allergy Clin. Immunol. 138(1), 107.e5-113.e5 (2016).

6 Lipworth BJ, Basu K, Donald HP et al. Tailored second-line therapy in asthmatic children with the Arg(16) genotype. Clin. Sci. 124(8), 521-528 (2013).

7 Vijverberg SJ, Pijnenburg MW, Hövels AM, Koppelman GH, Maitland-van der Zee AH. The need for precision medicine clinical trials in childhood asthma: rationale and design of the PUFFIN trial. Pharmacogenomics 18(4), 393-401 (2017).

8 Tonk ECM, Gurwitz D, Maitland-van der Zee AH, Janssens ACJW. Assessment of pharmacogenetic tests: presenting measures of clinical validity and potential population impact in association studies. Pharmacogenomics J. 17(4), 386-392 (2017).

9 Geenen JW, Baranova EV, Asselbergs FW, de Boer A, Vreman RA, Palmer CN et al. Early health technology assessments in pharmacogenomics: a case example in cardiovascular drugs. Pharmacogenomics 18(12), 1143-1153 (2017).

10 Manson LE, van der Wouden CH, Swen JJ, Guchelaar HJ. The Ubiquitous Pharmacogenomics consortium: making effective treatment optimization accessible to every European citizen. Pharmacogenomics 18(11), 1041-1045 (2017).

11 Wilffert B, Swen J, Mulder H, Touw D et al. From evidence based medicine to mechanism based medicine. Reviewing the role of pharmacogenetics. Int. J. Clin. Pharm. 35(3), 369-375 (2013).

12 van der Wouden CH, Swen JJ, Samwald M, Mitropoulou C, Schwab M, Guchelaar HJ. A brighter future for the implementation of pharmacogenomic testing. Eur. J. Hum. Genet. 24(12), 1658-1660 (2016).

13 Arwood MJ, Chumnumwat S, Cavallari LH, Nutescu EA, Duarte JD. Implementing pharmacogenomics at your institution: establishment and overcoming implementation challenges. Clin. Transl. Sci. doi:10.1111/cts.12404 (2016) (Epub ahead of print).

14 Verhoef TI, Ragia G, de Boer A et al. A randomized trial of genotype-guided dosing of acenocoumarol and phenprocoumon. $N$. Engl. J. Med. 369(24), 2304-2312 (2013).

15 van Schie RM, de Boer A, Maitland-van der Zee AH. Implementation of pharmacogenetics in clinical practice is challenging. Pharmacogenomics 12(9), 1231-1233 (2011). 
16 Gonzales D, Rao G, Bailey S et al. Precision dosing: public health need, proposed framework, and anticipated impact. Clin. Transl. Sci. 10, 443-454 (2017).

17 Puhan MA, Yu T, Stegeman I, Varadhan R, Singh S, Boyd CM. Benefit-harm analysis and charts for individualized and preference-sensitive prevention: example of low dose aspirin for primary prevention of cardiovascular disease and cancer. BMC Med. 13 , 250 (2015).

18 Bank PC, Caudle KE, Swen JJ et al. Comparison of the Guidelines of the Clinical Pharmacogenetics Implementation Consortium and the Dutch Pharmacogenetics Working Group. Clin. Pharmacol. Ther. 103(4), 599-618 (2017). 